\title{
Sistema de Información estudiantil para mejorar la gestión escolar del Centro Educativo Básico General EI Empalme
}

\section{Student Information System to improve the school management of the General Basic Education Center El Empalme}

\author{
Keren Hernández ${ }^{1}$, Edilsa Abrego ${ }^{1}$, Alcibíades Álvarez ${ }^{1}$, José Mendoza Avilés ${ }^{2}$ \\ ${ }^{1}$ Licenciatura en Desarrollo de Software - Centro Regional de Bocas del Toro - Universidad Tecnológica de Panamá. \\ ${ }^{2}$ Facultad de Ingeniería de Sistemas Computaciones - Centro Regional de Bocas del Toro - Universidad Tecnológica de Panamá.
}

Resumen En este trabajo se presenta, un sistema de información estudiantil para mejorar la gestión escolar del CEBG El Empalme. Se propuso un enfoque de investigación cualitativo, con un diseño no experimental, tipo transversal y descriptiva. La unidad de análisis es el CEBG El Empalme y la unidad de observación es un Sistema de Información estudiantil para mejorar la gestión escolar del CEBG El Empalme, distrito de Changuinola, Bocas del Toro, Panamá. En el proceso de desarrollo, se utilizó como instrumento la entrevista, la cual se enfocó en recolectar los datos pertinentes que se utilizaron dentro del sistema de gestión estudiantil para el CEBG El Empalme. El Sistema de Gestión Estudiantil se diseñó con una Metodología en Cascada: Framework Lineal, el mismo hace énfasis al entorno en el cual se plantea y estructura el desarrollo de un sistema. Se concluyó que, el sistema de gestión estudiantil cumplió con cada uno de los requisitos especificados para el desarrollo de la aplicación, la simplicidad del software permitió interactuar con el usuario y se adecuo a las necesidades del CEBG El Empalme.

Palabras clave Sistemas de información, gestión escolar.

\begin{abstract}
In this work we present a student information system to improve the school management of General Basic Education Center GBEC El Empalme. A qualitative research approach was proposed, with a non-experimental design, transversal and descriptive type. The unit of analysis is the GBEC El Empalme and the observation unit is a Student Information System to improve the school management of El Empalme GBEC, Changuinola district, Bocas del Toro, Panama. In the development process, the interview was used as an instrument, which focused on collecting the relevant data that was used within the student management system for the CEBG El Empalme. The Student Management System was designed with a Cascade Methodology: Linear Framework, which emphasizes the environment in which the development of a system is proposed and structured. It was concluded that, the student management system complied with each of the specified requirements for the development of the application, the simplicity of the software allowed interacting with the user and was adapted to the needs of the GBEC El Empalme.
\end{abstract}

Keywords Information system, system, school management.

*Corresponding author: jose.mendoza@utp.ac.pa

\section{Introducción}

Luego de la introducción de las Tecnologías de la Información y Comunicación (TIC) en la mayoría de los sistemas escolares del mundo, surge el interés por conocer qué se está haciendo con ellas, con qué fines y con qué impacto [1].

La evidencia hasta ahora muestra que el proceso de integración de las nuevas tecnologías al mundo escolar es menos fluido de lo esperado, presentando diversas barreras asociadas a las condiciones, prácticas y creencias existentes [1].
Otra consecuencia importante que han tenido las TIC en las escuelas es que son un instrumento muy potente para resolver viejas cuestiones históricas de la gestión escolar. En este punto conviven una preocupación de la escuela y una de la gestión de los sistemas educativos ligada con el uso de la información para la toma de decisiones. Una tendencia aún incipiente es que las escuelas no cuenten con plataformas en Internet [2].

En la actualidad, en Panamá el Ministerio de Educación posee diversos sistemas de información proporcionadas para gestionar la información de los estudiantes, por ejemplo, el Sistema de Administración de Centros Escolares (SIACE 2.0) 
[2], sin embargo, este sistema presenta inconvenientes y es algo complejo usar para ciertos docentes y administrativos.

Según [3], un sistema de información es un conjunto de componentes interrelacionados, con el objetivo de recolectar, procesar, almacenar y distribuir información para apoyar la toma de decisiones, la coordinación y el control de la organización.

Cabe destacar que dentro del sistema (SIACE 2.0) no existe una opción para llevar el control de la documentación del padre de familia o acudiente, en el proceso de matrícula, que entregan a los administradores para completar el expediente del alumno, además, no se lleva un control del total de la matrícula del centro educativo.

La administración y gestión educativa debe tener en cuenta también la penetración de las TIC en los hogares. De esta forma, no solo pueden establecerse nuevas interacciones y actividades en el seno de las familias, sino que abren un canal de comunicación entre la escuela y la familia que favorece la acción conjunta entre ambos agentes educativos [5].

Dadas las condiciones que anteceden se ha planteado la pregunta problema: ¿Cómo ayudaría un sistema de información estudiantil facilitar la gestión escolar del CEBG El Empalme?, ¿Cuáles serían los beneficios para el CEBG El Empalme?, y el objetivo del estudio es, desarrollar un sistema de información estudiantil que facilite la gestión escolar del CEBG El Empalme del Distrito de Changuinola.

En este mismo contexto el CEBG El Empalme cuenta con 73 grupos de estudiantes divididos entre preescolar, primaria y premedia. Actualmente enfrenta deficiencias con el sistema de información estudiantil, donde la mayoría de los datos de los acudidos son documentados llevados de manera manual y guardada en carpetas para posteriormente ser archivados.

Adicional a ello, los padres de familia apartan los cupos en el centro educativo, entregan parte de la documentación que solicitan, pero el estudiante no asiste formalmente en el año escolar, generando papelería gastada innecesariamente.

Cabe agregar que el sistema de gestión escolar es una estructura organizativa y relacional entre personas, que desempeñan las funciones, cantidad de personal directivo, docente y de servicios [2].

En ese mismo sentido, la creación de un sistema estudiantil facilitará la gestión de los cupos asignados a cada grupo, las inscripciones y un mejor control de la información de los estudiantes y profesores.

A pesar de lo anterior, hay algunos ejemplos de sistemas de gestión escolar que muestran que no es una cuestión de simplemente introducir las tecnologías en el mundo escolar y promover su uso, sino que los buenos resultados dependen de una serie de factores vinculados al contexto escolar, a las características de los profesores y modelos curriculares con que se trabajan [1].
Precisando de una vez, el Sistema de Información Estudiantil fue diseñado para brindar orden y simplicidad en la información redundante del CEBG El Empalme.

Además, el sistema se organizará en tres ideas principales:

- Características generales del diseño: las plataformas que requerirá el sistema, la modularización que tendrá y las restricciones y limitantes [3].

- Requisitos atendidos del diseño: requisitos funcionales o suplementarios que describirá las próximas secciones del sistema [4].

- Modelos y vistas que lo detallan: presenta al sistema como un todo, indicando en términos propios de la tecnología utilizada, las partes que lo forman y las relaciones principales entre ellas [5].

A manera de colofón, la finalidad de la investigación fue realizar un sistema de información estudiantil para mejorar la gestión escolar del CEBG El Empalme a través, de diversas técnicas para desarrollar un software de calidad, que contenga diversas funciones para agilizar el proceso de control de datos. Asimismo, se caracterizó por la recolección de datos mediante un gestor de base de datos que contará el programa construido con varios requisitos que ayudaron a precisar la información de manera más detallada, clara y eficiente [6].

\section{Materiales y Métodos}

\subsection{Tipo y diseño de estudio}

La investigación posee un enfoque cualitativo, porque se utilizó la recolección de datos sin medición numérica, para descubrir o afinar las preguntas de investigación en el proceso de interpretación, de modo que el proyecto de investigación busca principalmente optimar los procesos y llevar a cabo el control de los datos [7].

Por otra parte, de acuerdo con sus características, el diseño de investigación es no experimental, ya que se basa en la elaboración y desarrollo de un software para solucionar las necesidades tecnológicas de un centro educativo, y en efecto, se basó en la observación, profundidad de los datos, detalles y experiencias únicas en el contexto natural para luego ser analizados [7].

Es evidente entonces que el tipo de investigación es transversal, en donde, se recolectan los datos en un solo momento, en un único tiempo por medio de técnicas de estudio para luego analizarlos. En igual forma se define la investigación de manera descriptiva porque mide la información de manera conjunta presentando la importancia de cualquier fenómeno o variable que se analiza [7].

Como resultado el tipo de investigación es prospectiva, puesto que la información es de primera mano a medida que va sucediendo la investigación.

Cada afiliación debe incluir los siguientes elementos: unidad a la que está adscrito, entidad a la que está afiliado y el correo electrónico. El último elemento es obligatorio porque facilita la comunicación entre el autor y el lector. 


\section{2 Área de estudio}

La investigación se realizó en el Distrito de Changuinola en el Centro Educativo Básico General (CEBG) El Empalme, ubicado en la Ave. Omar Torrijos 164, en las siguientes coordenadas: $9^{\circ} 26^{\prime} 20.4^{\prime \prime} \mathrm{N} 82^{\circ} 31^{\prime} 16.1 " \mathrm{~W}$ (figura 1).

\subsection{Unidad de análisis y observación}

Tal como se ha visto, la unidad de análisis es el CEBG El Empalme y la unidad de observación es un Sistema de Información estudiantil para mejorar la gestión escolar del CEBG El Empalme.

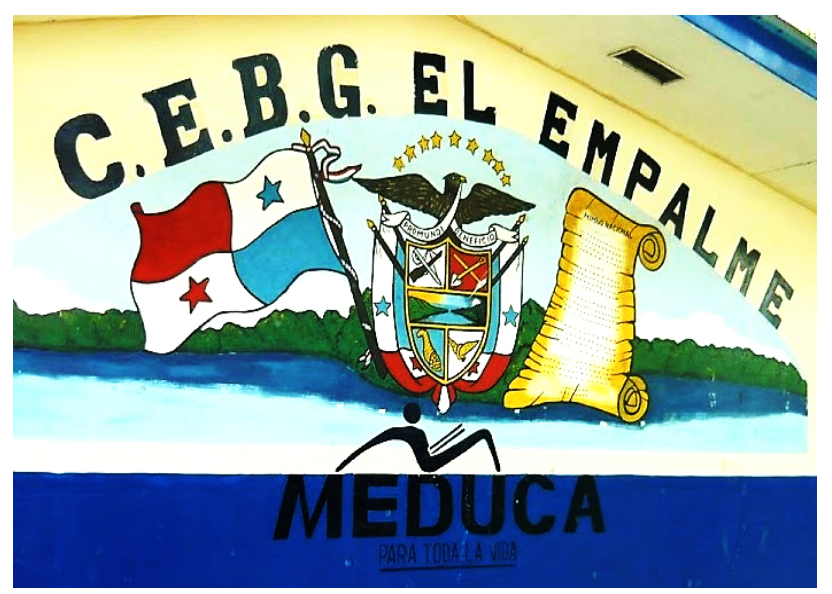

Figura 1. Centro Educativo Básico General El Empalme.

\subsection{Población y muestra}

El Centro Educativo cuenta con un total de 2,366 estudiantes entre Preescolar, Primaria y Premedia y con 33 profesores y 55 docentes de grado.

La población estudiantil se divide en tres niveles: así como lo muestra la siguiente tabla 1 :

Tabla 1. Matrícula del CEBG El Empalme [8]

\begin{tabular}{lcc}
\hline Preescolar & Cantidad de Grupos & Matrícula por Grupo \\
\hline Preescolar & 6 & 30 \\
\hline Primaria & Cantidad de Grupos & Matrícula por Grupo \\
\hline 1er grado & 8 & 30 \\
2do grado & 8 & 30 \\
3er Grado & 7 & 30 \\
4to grado & 7 & 30 \\
5to grado & 8 & 30 \\
6to grado & 7 & 30 \\
\hline Premedia & Cantidad de Grupos & Matrícula por Grupo \\
\hline 7mo Grado & 9 & 38 \\
8vo Grado & 8 & 38 \\
8no Grado & 7 & 38 \\
\hline
\end{tabular}

Se puede resaltar que la matrícula actual del CEBG El Empalme con relación a preescolar es de 8\%, primaria es de $57 \%$, donde abarca la mayor cantidad de matrícula y premedia
$35 \%$ menos de la mitad que primaria, tal como se aprecia en la figura 2 .

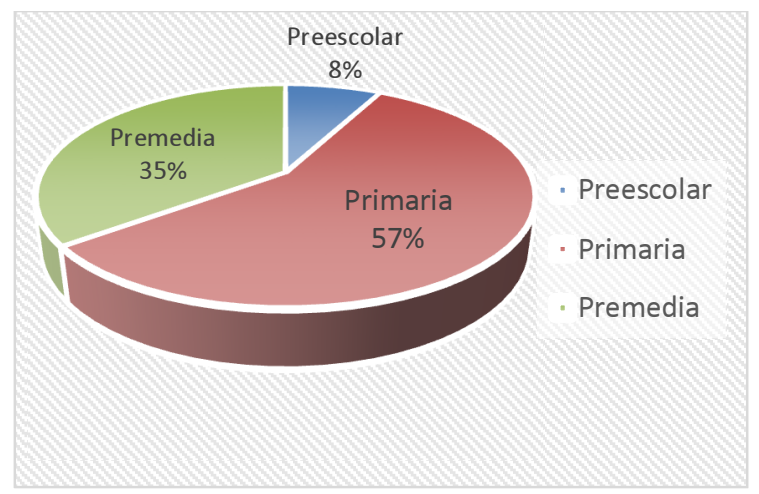

Figura 2. Matrícula del año 2018 del CEBG El Empalme.

Sin embargo, la cantidad de estudiantes y salones es cambiante durante el inicio del periodo escolar, ya que varía la demanda en algunas ocasiones en primaria o preescolar, debido a esta exigencia, se toman decisiones de abrir aulas nuevas o tener que ingresar hasta 40 estudiantes por salón. Con respecto a premedia, se mantiene la cantidad de estudiantes.

Para este estudio, no se aplica el muestreo porque se desarrolló un software para estudiar la población estudiantil y gestionar la información de los estudiantes, padres de familia, profesores, maestros y administrativos.

\subsection{Variables}

La gestión escolar es la variable estudiada, la cual es un proceso que enfatiza la responsabilidad del trabajo en equipo e implica la construcción, diseño y evaluación del quehacer educativo, para la ejecución y el monitoreo de los mecanismos, las acciones y las medidas necesarias para la consecución de los objetivos de la institución [8].

El sistema de información se realizó mediante dos entornos:

- El entorno de la aplicación: implicará cómo será la interfaz del software que se realizó, contará de requisitos suplementarios tales como:

- Simplicidad: medirá si el desplazamiento del sistema es sencillo.

- Adecuación: evaluará que el sistema cumple con los requisitos del Centro Educativo El Empalme (CEBG).

- Confiabilidad: determinará el nivel de protección en relación con el acceso a partes específicas del sistema.

- Usabilidad de la aplicación: comprobará si el sistema es amigable o tiene facilidad de uso para los usuarios.

\subsection{Métodos e instrumentos de recolección de datos}

Sobre la recolección de datos [7] explica que, "una vez que, seleccionamos el diseño de investigación apropiado, de acuerdo con el problema de estudio, la siguiente etapa consiste 
en recolectar los datos pertinentes sobre los atributos, conceptos o variables de las unidades de análisis o casos".

En el proceso de desarrollo se utilizó como instrumento la entrevista, la cual se enfocó en cómo desarrollar un sistema de gestión estudiantil para el CEBG El Empalme. Este instrumento ayudó a recolectar los datos pertinentes que se utilizó dentro del sistema y a la realización de la evaluación del software.

Cabe resaltar que en la entrevista se tomaron en cuenta dos factores como la gestión estudiantil y la población estudiantil para el desarrollo de software.

Las preguntas de la entrevista fueron validadas por un panel de expertos en redacción, además, la validación en la propuesta se refiere a la comprobación final de las etapas de vida del desarrollo, de que el producto creado satisface correctamente la especificación de requisitos pedidos y las expectativas que han depositado en el mismo [9].

Al mismo tiempo, se validó el software para comprobar, si el producto obtenido cumple los requisitos del administrador [9].

Esta actividad debe realizarse en las áreas del ambiente de operación, en donde se tomaron en cuenta las observaciones presentadas para mejorar la misma, por especialistas en software de la Universidad Tecnológica de Panamá, que registra los resultados. De acuerdo con las observaciones dadas por los especialistas en software de la Universidad Tecnológica de Panamá, el cual muestra la tabla 2, el Sistema de Información Estudiantil, muestra un $60 \%$ de validación del resultado esperado. Esto se debe a que el sistema está contemplado para mejoras en el futuro. el cual muestra la siguiente tabla 2.

Tabla 2. Sistema de Información Estudiantil para mejorar la gestión escolar del CEBG El Empalme

\begin{tabular}{|l|r|r|r|}
\hline Preguntas & Excelente & Bueno & Malo \\
\hline Simplicidad & & $5 \%$ & \\
\hline Adecuación & & $5 \%$ & \\
\hline Confiabilidad & $20 \%$ & & \\
\hline Usabilidad & & $5 \%$ & \\
\hline Diseño del Sistema & & $5 \%$ & \\
\hline Mantenimiento & & & \\
\hline Validación & & $10 \%$ & \\
\hline Total: & $\mathbf{6 0 \%}$ verificar & \\
\hline
\end{tabular}

A continuación, se procede a definir cada una de ellas:

- Confiabilidad: Habilidad que tiene el sistema para realizar funciones bajo condiciones específicas.

- Diseño de sistema: Desarrollo de directrices durante el proceso del software para satisfacer los objetivos planteados desde el punto de vista funcional.
- Entorno gráfico: Conjunto de programas que ofrecen al usuario un entorno visible más amigable.

- Rapidez: Capacidad de computación y consumo de funcionamiento del sistema.

- Codificación: Personas que trabajan juntas en una estación de trabajo con el objeto de crear código de calidad para la resolución de un problema.

- Mantenimiento: Es una medida en el cual el sistema realiza reparaciones y mantenimiento a la Base de Datos, para seguir optimizando los procesos.

- Pruebas: Verificación de los procesos que se realizan dentro del sistema.

- Validación: Al lenguaje de programación o las herramientas automatizadas [6].

Esta valoración busca corroborar la conformidad del producto software con respecto a los requisitos funcionales y no funcionales, como se muestra en la figura 3.

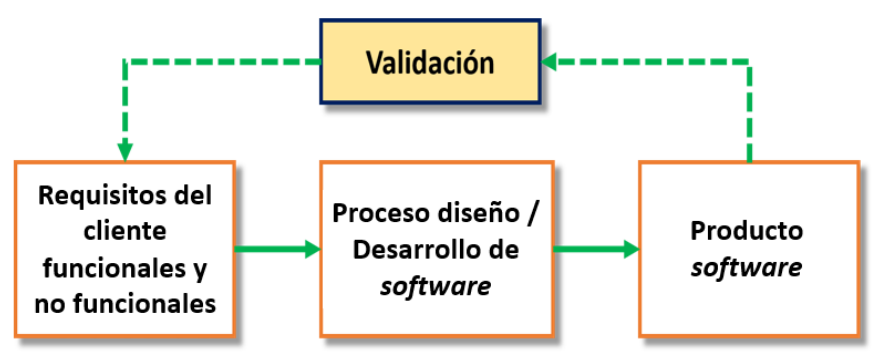

Figura 3. Diagrama para la validación. Fuente: [14].

La evaluación de los especialistas de la Facultad de Ingeniería de Sistemas Computacionales, de la Universidad Tecnológica de Panamá, Centro Regional de Bocas del Toro, certifica que el programa cumple con cada uno de los requisitos del cliente.

La entrevista fue aplicada el día 23 de abril del 2018, en el CEBG El Empalme, al director del Centro Educativo el Empalme.

Naturalmente este diseño se ha ajusto a las necesidades y requisitos dados por el Administrador del CEBG, de manera que los datos se recogieron por medio de entrevistas para facilitar el desarrollo del software.

\section{Resultados y Discusión}

Como se ha mencionado anteriormente, el Sistema de Gestión Estudiantil se diseñó con una metodología en cascada: Framework Lineal, el mismo hace énfasis al entorno en el cual se plantea y estructura el desarrollo de un sistema.

La metodología en cascada es un proceso de desarrollo secuencial, en el que el desarrollo se ve fluyendo hacia abajo, como una cascada, sobre las fases que componen el ciclo de vida [13]. 
Cabe señalar que, el Framework Lineal es el enfoque metodológico que ordena rigurosamente las etapas del proceso para el desarrollo de software, de tal forma que el inicio de cada etapa debe esperar a la finalización de la etapa anterior.

La aplicación se desarrolló en varias etapas como lo muestra la figura 4.

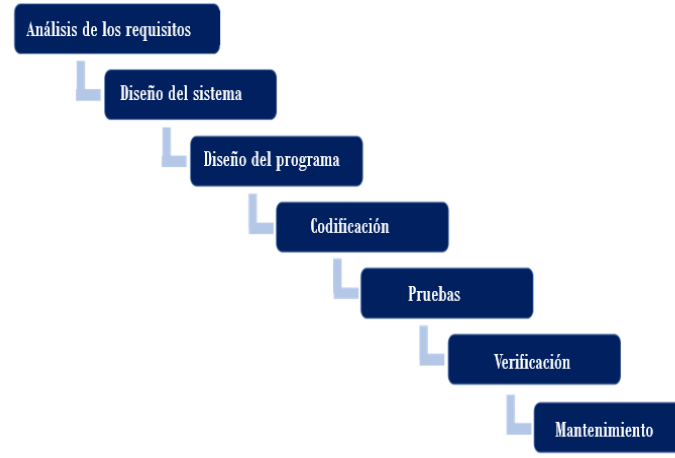

Figura 4. Fases de desarrollo. Fuente: Elaboración propia a partir de [11].
Como se indicó, el gestor de base de datos que se utilizó fue $S Q L$ Server, sistema de gestión de bases de datos relacionales de Microsoft [12], que está diseñado para el entorno empresarial, donde se mostró las relaciones que hay entre las tablas, los atributos y métodos que tendrán cada una de ellas, así como lo muestra la siguiente figura 5 .

El Sistema de Gestión Educativa se aplicó a los usuarios y administrador es del CEBG El Empalme, donde el sistema gestione:

- Registro de roles y privilegios.

- Cupos asignados por grupo (con un aproximado de 35-40 estudiantes).

- Formulario de preinscripciones.

- Formulario de inscripción.

- Control de información.

- Asignar a los profesores y maestros a cada grupo.

- Distribuir a los estudiantes por consejería.

- Búsqueda de los estudiantes.

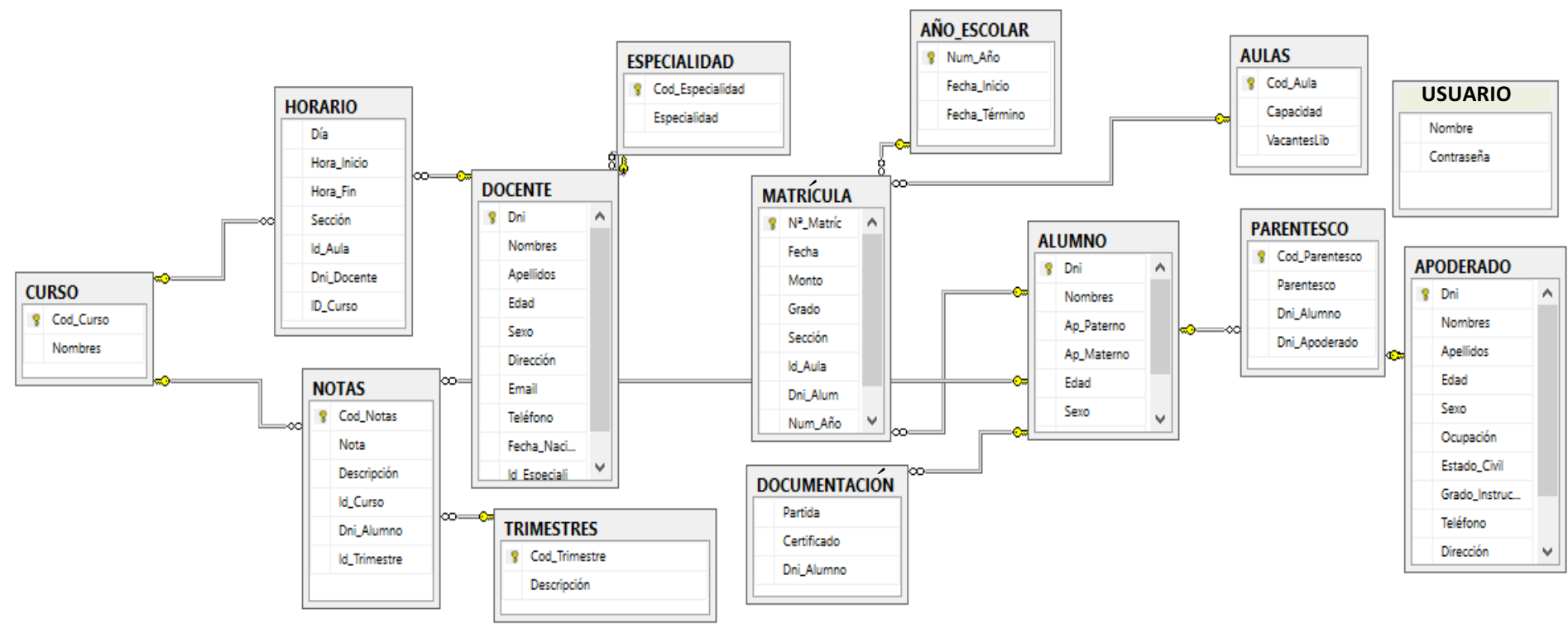

Figura 5. Diagrama de base de datos.

El análisis de requisitos o módulos se desarrolló de la siguiente manera:

- Login: Introducir el usuario y la contraseña.

- Menú principal: Muestra el panel de opciones a elegir.

- Módulo alumnos: Busca el registro de alumnos y agrega alumnos.

- Formulario acudiente: Muestra la opción de registrar o consultar el acudiente.

- Buscador: Consulta al estudiante, muestra la información de este.
- Listado de aulas: Selecciona las aulas que existen en el Colegio, la capacidad que tiene cada salón y la cantidad de estudiante que tiene.

- Registro de año escolar: Programa el año escolar y define la fecha del inicio y final del año escolar.

- Preinscripción: Muestra la opción de registrar o consultar la matrícula de los alumnos.

- Registro de inscripción: Registra los alumnos que serán matriculados en el año escolar.

- Formulario docente: Muestra la opción de registrar y ver horarios de docentes. 
Hernández (et al): Sistema de Información estudiantil para mejorar la gestión escolar del Centro Educativo Básico General El Empalme

La aplicación contó con tres diferentes tipos de niveles de perfil:

- Nivel 1: Nivel administrador, es el encargado de tener el control único de eliminación, inserción, modificación, consulta y actualización de todas las ventanas del software.

- Nivel 2: Nivel usuario, solo tiene acceso a consultar e insertar los profesores y maestros del plantel.

- Nivel 3: Nivel capturador, en este nivel solo se podrá captar los datos de los acudientes y acudidos al momento de la realización de la preinscripción e inscripción.

El tipo de seguridad de la aplicación es tradicional, con contraseñas para los tres tipos de niveles diferentes, además el Sistema de Gestión Educativo se desarrolló en la v 1.0.

Como es sabido, un programa utilizado en un entorno del mundo real está destinado a cambiar, es por ello, que al administrador del Sistema de Gestión Estudiantil se le brindó la propuesta de mejoras en un futuro próximo.

El Sistema de Gestión Educativa del CEBG El Empalme, o bien como se llamó SIS MATR (Sistemas de Matrícula), se realizó en la v1.0. A continuación se muestra unas de las principales pantallas del SIS MATR v1.0.

Inicio de sesión: Permitió al administrador iniciar sesión ingresando el nombre de usuario y la contraseña, para luego dar clic en "Entrar" permitiendo acceso al SIS. MATR, al dar clic al botón de salir el sistema automáticamente se cierra como lo muestra la siguiente figura 6 .

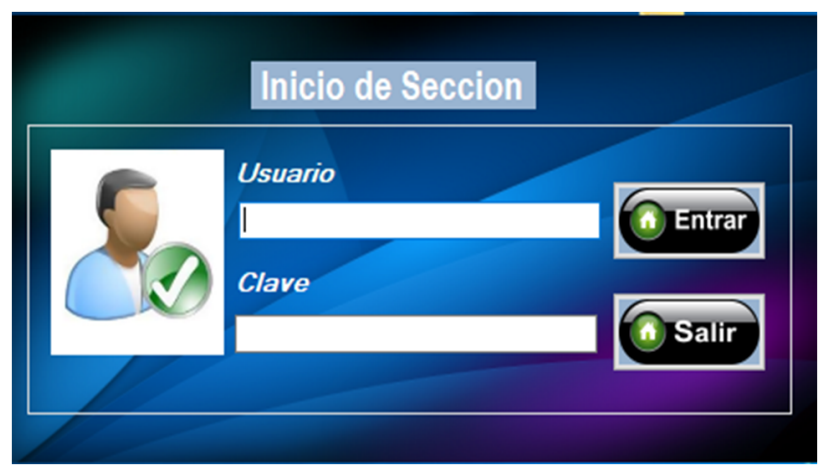

Figura 6. Inicio de sesión.

Menú Principal: Despliega la pantalla principal, donde muestra los botones de alumnos, acudientes, docente, aulas, matricula, año escolar, la fecha y hora actual, al hacer clic en las siguientes opciones el sistema inmediatamente mostrará otra pantalla, así como los muestra la figura 7.

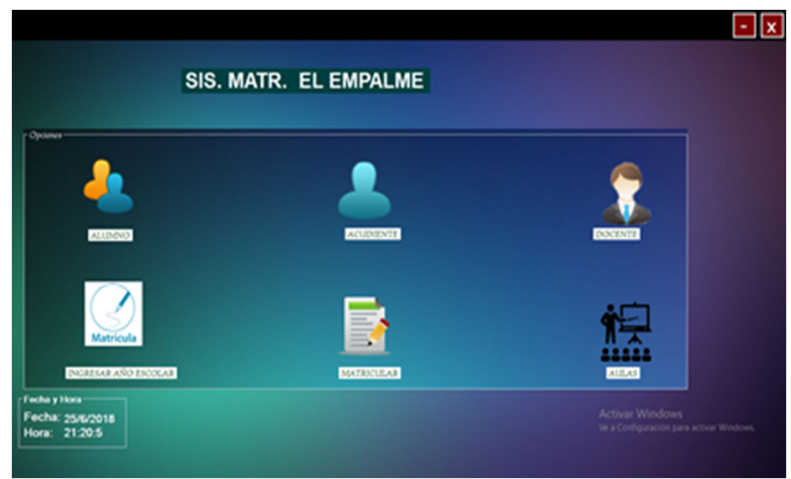

Figura 7. Menú Principal.

Registro de alumno: Permite registrar a los estudiantes que ingresan por primera vez al Centro Educativo Básico General El Empalme y a su vez, buscar a un alumno por el número de identidad personal si ya existe, así como lo muestra la figura 8 . Es importante destacar que todos los campos son obligatorios para el registro del estudiante

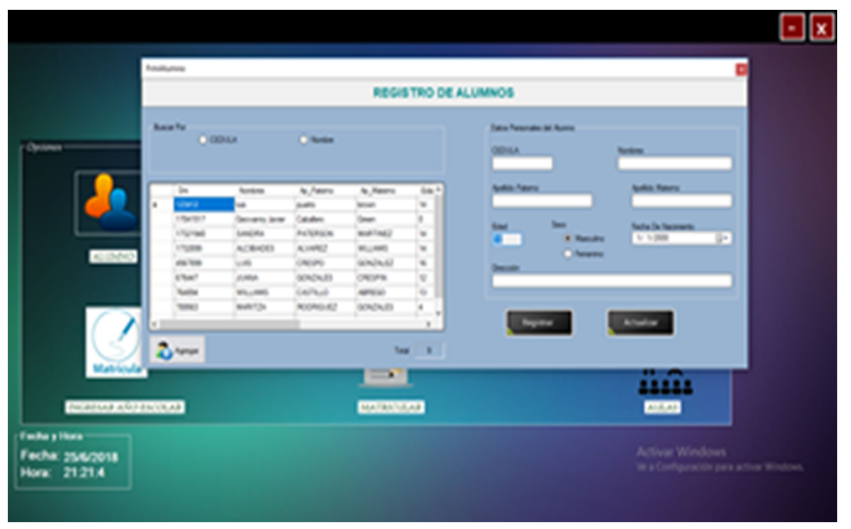

Figura 8. Registro alumno.

Registro y búsqueda de acudiente: En esta sección se requiere toda la información del acudiente (campos obligatorios), el usuario debe ingresar al acudiente que está inscribiendo al estudiante como muestra la figura 9.

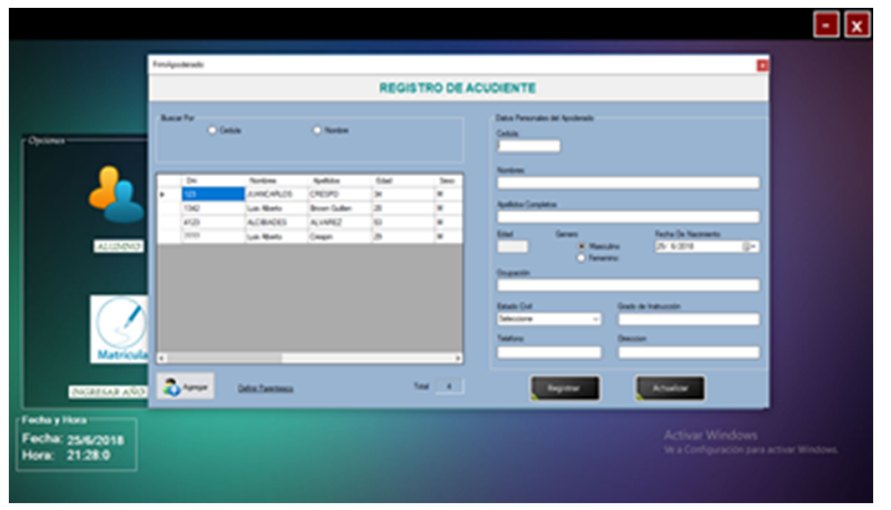

Figura 9. Registro y búsqueda acudiente. 
Registro de docente: Datos personales, formación académica y perfil profesional, etc. Una vez obtenidos todos los datos se procede a guardar la información introducida en la interfaz de docente como se ve en la siguiente figura 10.

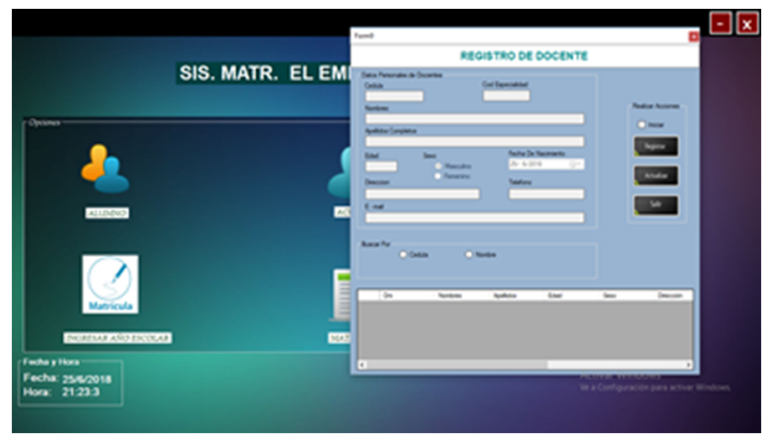

Figura 10. Registro docente.

Registro de Año Escolar: Al hacer clic se despliega el formulario donde se ingresa el proceso de principio del año escolar hasta la culminación del periodo escolar actual. Al presionar clic en Registrar, se podrá observar que se guardó el periodo de año escolar como podemos observar en la figura 11.

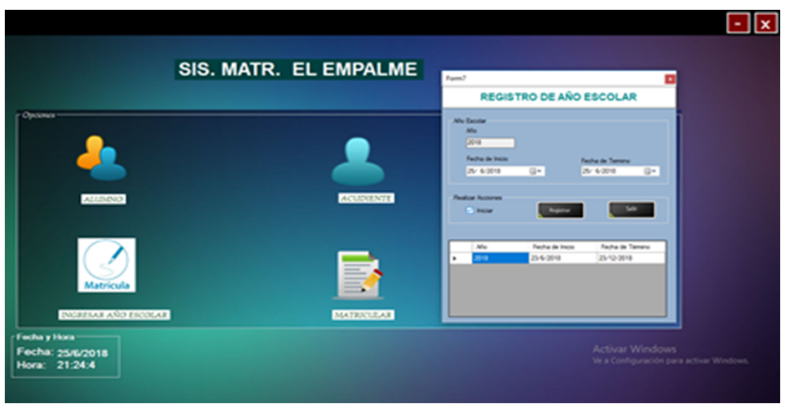

Figura 11. Registro de año escolar.

Registro de aulas: Esta opción permite adicionar, actualizar y consultar aulas del centro y la cantidad de estudiantes que hay en un salón. Al ingresar a esta opción se encuentra la pantalla de disponibilidad de vacantes disponibles, así como se muestra en la figura 12 .

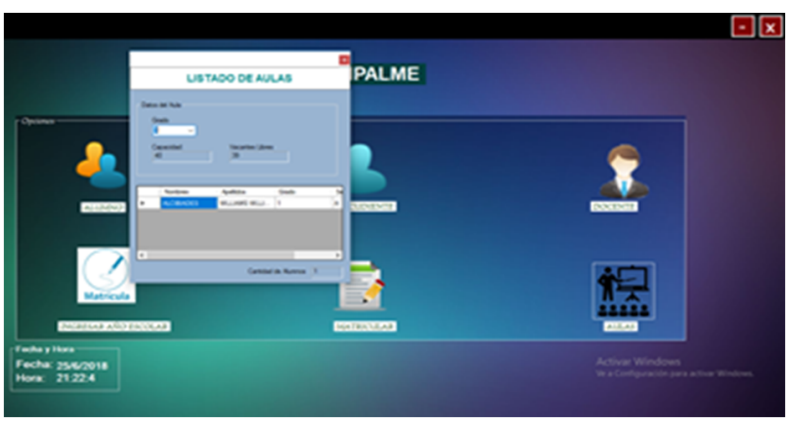

Figura 12. Registro aulas.
Consulta de matrícula: Dentro de la pantalla de matrícula se encuentra el registro de los estudiantes ya matriculados, que es de vital importancia para el manejo de cupos. Una vez ingresada, se oprime el botón Buscar, con lo cual aparecerá la siguiente pantalla como se observa en la figura 13.

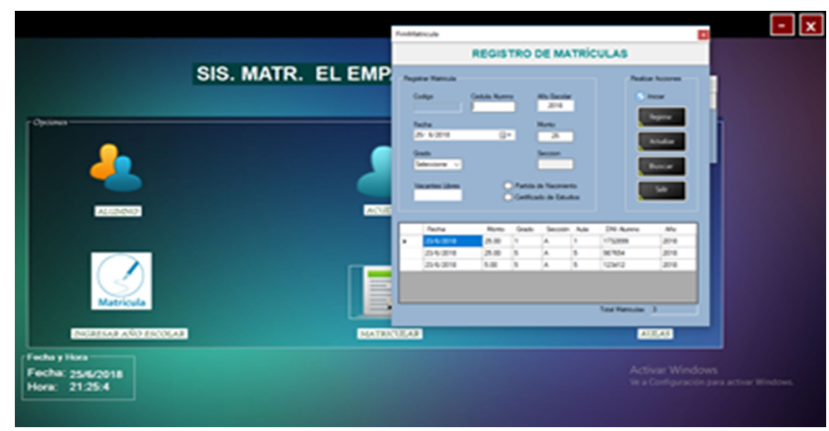

Figura 13. Consulta Matricula.

\section{Conclusiones}

De acuerdo con la entrevista realizada al administrador del CEBG El Empalme, el sistema de gestión estudiantil cumplió con cada uno de los requisitos especificados para el desarrollo de la aplicación, la simplicidad del software permitió interactuar con el usuario y se adecuo a las necesidades del CEBG El Empalme sin complejidad.

Asimismo, se caracterizó por la recolección de datos mediante un gestor de base de datos que contará el programa construido con varios requisitos que ayudaron a precisar la información de manera más detallada, clara y eficiente.

Entre sus ventajas está la creación de un sistema estudiantil que facilitó la gestión de cupos asignados a cada grupo, las inscripciones y control de la información de los estudiantes y profesores.

Dos especialistas de la UTP Centro Regional de Bocas del Toro evaluaron la aplicación del software, indicando que es un programa útil para el avance de la educación en la provincia de Bocas del Toro.

Es importante recalcar que este proyecto está en proceso de mejoras.

\section{AGRADECIMIENTO}

Agradecemos a la Jornada de Iniciación Científica y a la Universidad Tecnológica de Panamá, por contribuir a la formación profesional; al profesor asesor, Dr. José Mendoza, por impartir sus conocimientos siempre en cada etapa del artículo de investigación; y a la Dra. Vanessa Valdés, por su apoyo incondicional, al director del CEBG El Empalme, profesor Armando Boniche, por proporcionar la información para realizar la investigación. 
Hernández (et al): Sistema de Información estudiantil para mejorar la gestión escolar del Centro Educativo Básico General El Empalme

\section{REFERENCIAS}

[1] M. Claro, "La incorporación de tecnologías digitales en educación. Modelos de identificacion de buenas practicas," CEPAL, p. 30, 2010.

[2] J. C. Tedesco and D. Iglesias, "La integración de las Tecnologías de la Información y la Comunicación en los Sistemas Educativos," 2006. [Online]. Available: http://unesdoc.unesco.org/images/0015/001507/150785s.pdf. [Accessed 208 2018].

[3] MEDUCA, "Manual de usuario de SIACE," 126 2009. [Online]. Available: http://www.meduca.gob.pa/sites/default/files/201512/SistemaAdministracionCentrosEscolares.pdf. [Accessed 58 2018].

[4] K. Laudon and J. Laudon, Sistemas de Información Gerencial, 12 ed., México: Pearson Education, 2012, p. 640.

[5] R. Carneiro, J. C. Toscano and T. Díaz, "Los desafíos de las TIC para el cambio educativo," Fundación Santillana, Madrid, 2011.

[6] N. Rodríguez, "Gestión Escolar y la Calidad de Enseñanza," EDUCARE, vol. IV, no. 10, p. 9.

[7] M. Arias Chaves, "La ingeniería de requerimientos y su importancia en el desarrollo de proyectos de software," InterSedes (C) Universidad de Costa Rica, vol. VI, no. 10, pp. $2-$ 4, 2005

[8] P. Pytel, H. D. Ramon, M. F. Pollo Cattaneo, P. V. Britos and R. Garcia Martínez, "Ingeniería de requisitos basada en técnicas de ingeniería del conocimiento," SEDICI, pp. 426-429, 2011.

[9] L. E. Mendoza , M. A. Pérez and A. C. Griman, "Prototipo de Modelo Sistémico de Calidad (MOSCA) del Software," Scielo, vol. VIII, no. 3, pp. 196-227, 2005.
[10] R. S. Pressman, Ingenieria de Software, septima ed., P. R. Vázquez, Ed., México, D. F.: McGRAW-HILL INTERAMERICANA EDITORES, S.A., 2010.

[11] R. Hernández Sampieri, C. Fernández Collado and P. Baptista Lucio, Metodología de la Investigación, Cuarta ed., Mexico: Mc Graw Hill, 2006.

[12] A. Boniche, Interviewee, Director del CEBG El Empalme, [Interview]. 2042018.

[13] G. Ruíz, "Gestión Institucional: conceptos introductorios," Educ.ar, pp. 1-7, 2007.

[14] J. Blanco LLano and A. Rodríguez Hernández, "Revisón, Verificación y Validación en un proceso de Desarrollo de Software," Redalyc, vol. XXXII, no. 1, pp. 28-36, 3 Enero 2011.

[15] M. Herrera, "Ingeniería del software: Metodologías y ciclos de vida," $32009 . \quad$ [Online]. Available: http://www.academia.edu/9795641/INGENIER\%C3\%8DA_DE L_SOFTWARE_METODOLOG\%C3\%8DAS_Y_CICLOS_DE _VIDA_Laboratorio_Nacional_de_Calidad_del_Software.

[16] Y. Bassil, "A Simulation Model for the Waterfall Software Development Life Cycle," International Journal of Engineering \& Technology, vol. 2, no. 5, 552012.

[17] Microsoft, "Microsoft SQL Server," 2017. [Online]. Available: https://www.microsoft.com/es-mx/sql-server/sql-server-2017. [Accessed 58 2018]. 